УДК 004.94

\title{
АНАЛИЗ ЭВОЛЮЦИИ ДИНАМИЧЕСКОЙ СИСТЕМЫ ТИПА ОТОБРАЖЕНИЯ РЁССЛЕРА
}

\section{Осипов Геннадий Сергеевич} д.т.н., зав кафедрой информатики Вашакидзе Нателла Семеновна Филиппова Галина Викторовна Рауш Наталья Леонидовна старшие преподаватели ФГБОУ ВО «Сахалинский государственный университет»

Аннотация: в терминах современного формально-математического аппарата изложены основы методологии качественного анализа эволюции для серии динамических диссипативных систем Рёсслера со странным аттрактором.

Компьютерное моделирование, количественный анализ и практическая апробация теоретических предпосылок выполнены в среде пакета компьютерной алгебры Wolfram Mathematica.

Моделирование выполнено для различных экспериментов при широком спектре варьируемых параметрах исследуемых моделей. Предложена концепция визуализация результатов анализа модели в виде интегральных и фазовых траекторий как на плоскости, так и в пространстве.

Ключевые слова: динамическая система Рёсслера, исследование на устойчивость, компьютерное моделирование. 


\title{
ANALYSIS OF THE EVOLUTION OF A DYNAMIC SYSTEM OF THE RESSLER MAPPING TYPE
}

\author{
Osipov Gennady Sergeyevich \\ Vashakidze Natella Semyonovna \\ Filippova Galina Viktorovna \\ Rausch Natalia Leonidovna
}

\begin{abstract}
In terms of the modern formal mathematical apparatus. the fundamentals of the methodology of qualitative analysis of evolution for a series of dynamic dissipative Ressler systems with a strange attractor are presented.

Computer modeling, quantitative analysis, and practical testing of theoretical assumptions are performed in the environment of the Wolfram Mathematica computer algebra package.

The simulation was performed for various experiments with a wide range of variable parameters of the models under study. The concept of visualization of the results of the model analysis in the form of integral and phase trajectories both on the plane and in space is proposed.
\end{abstract}

Key words: Ressler dynamic system, stability study, computer simulation.

\section{Введение.}

Инновационное развитие методологии современных исследований в области моделирования очень сложных диссипативных систем базируется, в основном, на конвергентных парадигмах сочетания разносторонних подходов к фундаментальному анализу, нацеленному на достижение единой цели построению модели исследуемых структур как для реализации их априорного синтеза, так и перспективного управления и оптимизации.

В настоящее время проявляется интерес к исследованию сложных динамических систем со странными аттракторами, т.к. они «присутствуют» во многих естественных диссипативных системах, например типа фондовых бирж, для которых принципиально не подтверждается «гипотеза эффективного рынка» и которые подвержены эффекту постепенного рассеивания и поглощения внутренней и поступающей внешней энергии (информации).

Аттрактор Рёсслера наблюдается во многих системах. Например, он применяется для описания потоков жидкости, а также при изучении поведения 
различных химических реакций и молекулярных процессов. Впервые простую трёхмерную модель динамики химических реакций, протекающих в некоторой смеси с перемешиванием, предложил практикующий врач Отто Рёсслер (1976). [1].

\section{1. Формальная постановка задачи.}

Исследуется неоднородная нелинейная автономная динамическая система Рёсслера, которая в классической постановке имеет вид [1]:

$$
\left\{\begin{array}{l}
\frac{d x}{d t}=-(y+z) \\
\frac{d y}{d t}=x+a y \quad(a, c>0 ; b \geq 0) \\
\frac{d z}{d t}=b+z(x-c)
\end{array}\right.
$$

В системе имеются две стационарные точки:

$$
E_{1,2}(\bar{x}, \bar{y}, \bar{z})=E_{1,2}\left(\frac{c \mp \sqrt{c^{2}-4 a b}}{2}, \frac{-c \pm \sqrt{c^{2}-4 a b}}{2 a}, \frac{c \mp \sqrt{c^{2}-4 a b}}{2 a}\right) \text {. }
$$

Очевидно, $\left\{\begin{array}{l}\bar{x}=-a \bar{y} \\ \bar{z}=-\bar{y}\end{array}\right.$.

Целью исследования является качественная формализация эволюции и количественное (численное, компьютерное) моделирование трех модификаций системы Рёсслера, от ее базового варианта:

$$
\left\{\begin{array}{l}
\frac{d x}{d t}=-(y+z) \\
\frac{d y}{d t}=x+a y \quad(a, c>0) \\
\frac{d z}{d t}=z(x-c)
\end{array}\right.
$$

и до аналитического обобщения в виде:

$$
\left\{\begin{array}{l}
\frac{d x}{d t}=-(y+z) \\
\frac{d y}{d t}=x+a y \quad(a, b, c>0) \\
\frac{d z}{d t}=b x+z(x-c)
\end{array}\right.
$$




\section{2. Моделирование базовой системы.}

Очевидно, в системе (2) присутствуют две стационарные точки:

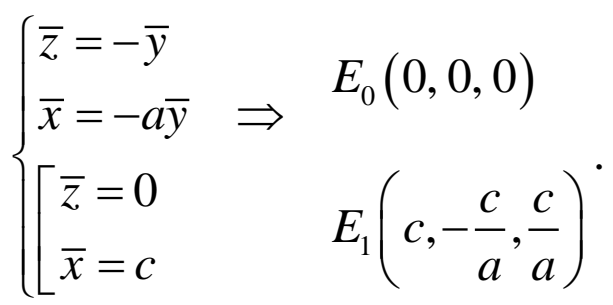

2.1. Исследование поведения системы в окрестности нулевой стационарной точки $E_{0}(0,0,0)$

2.1.1. Основы качественного анализа

В данном случае $\bar{x}_{0}=\bar{y}_{0}=\bar{z}_{0}=0$ и система (2) после линеаризации в окрестности данной точки преобразуется к виду:

$$
\left\{\begin{array}{l}
\frac{d \tilde{x}}{d t}=-(\tilde{y}+\tilde{z}) \\
\frac{d \tilde{y}}{d t}=\tilde{x}+a \tilde{y} \\
\frac{d \tilde{z}}{d t}=-c \tilde{z}
\end{array},\right.
$$

где $\tilde{x}, \tilde{y}, \tilde{z}$-- малые приращения соответствующих координат.

Соответственно определится Якобиан:

$$
J_{0}=\left(\begin{array}{ccc}
0 & -1 & -1 \\
1 & a & 0 \\
0 & 0 & -c
\end{array}\right)
$$

с параметрами $\left|J_{0}\right|=-c ; \operatorname{tr}\left(J_{0}\right)=a-c$.

Построение и решение характеристического уравнения позволяют получить значения трех собственных чисел:

$$
\lambda^{3}+(c-a) \lambda^{2}+(1-a c) \lambda+c=0 \Rightarrow\left[\begin{array}{l}
\lambda_{1}=-c \\
\lambda_{2,3}=\frac{a \pm \sqrt{a^{2}-4}}{2}
\end{array}\right.
$$

\subsection{2. Компьютерное моделирование}

На рисунке 1 представлен начальный фрагмент фазовой траектории системы при $a=0.2, c=0.5$ в трехмерном пространстве. Из точки близкой к стационарной $E_{0}(0,0,0)$ траектория начинает раскручиваться по спирали, причем координата $z$ остается практически неизменной. 


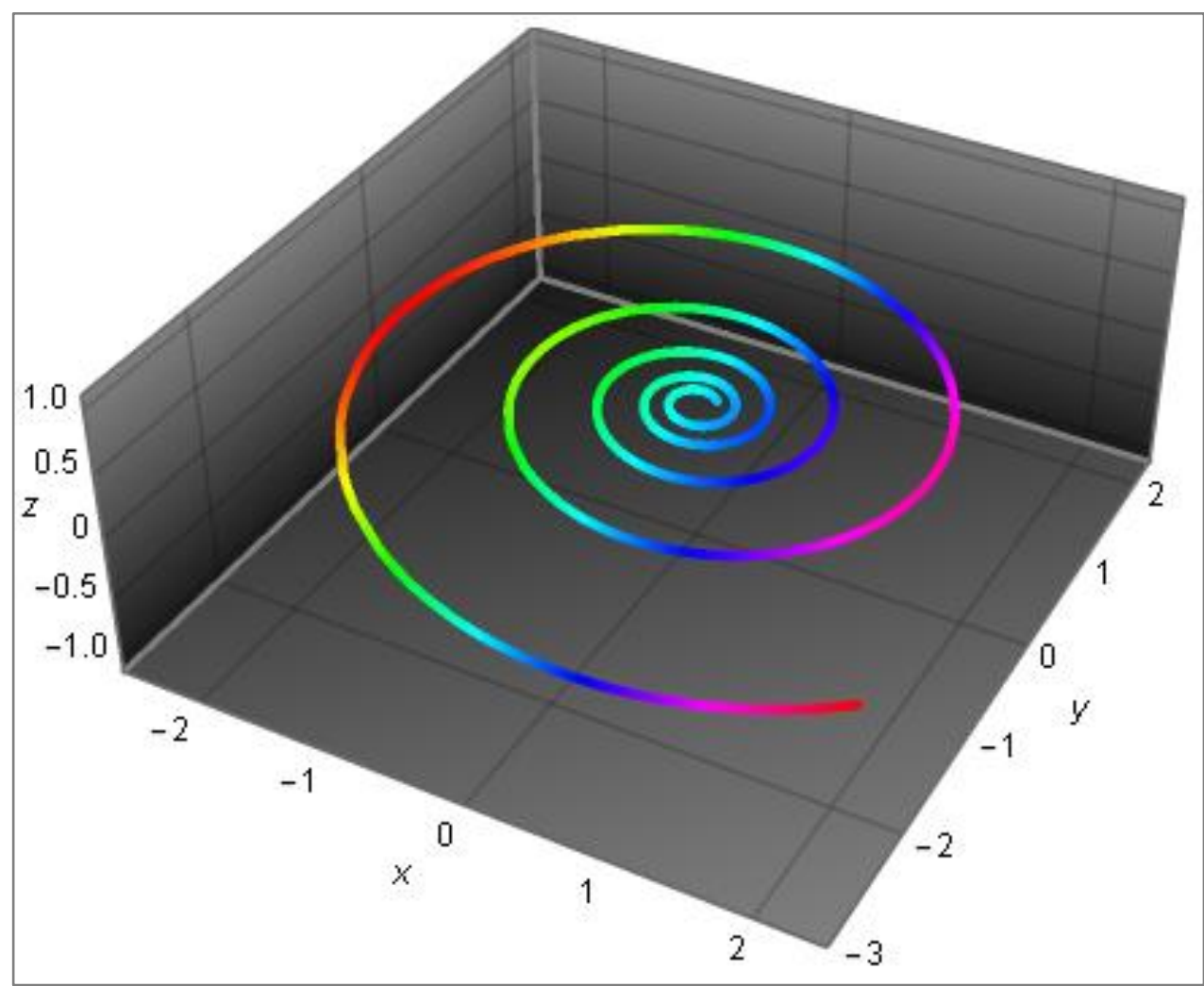

Рис. 1. Фазовое пространство

Решения системы $x=x(t), y=y(t), z=z(t)$ представлены на рисунке (2). Координаты $x$ и $y$ достаточно быстро расходятся от стационарной точки.

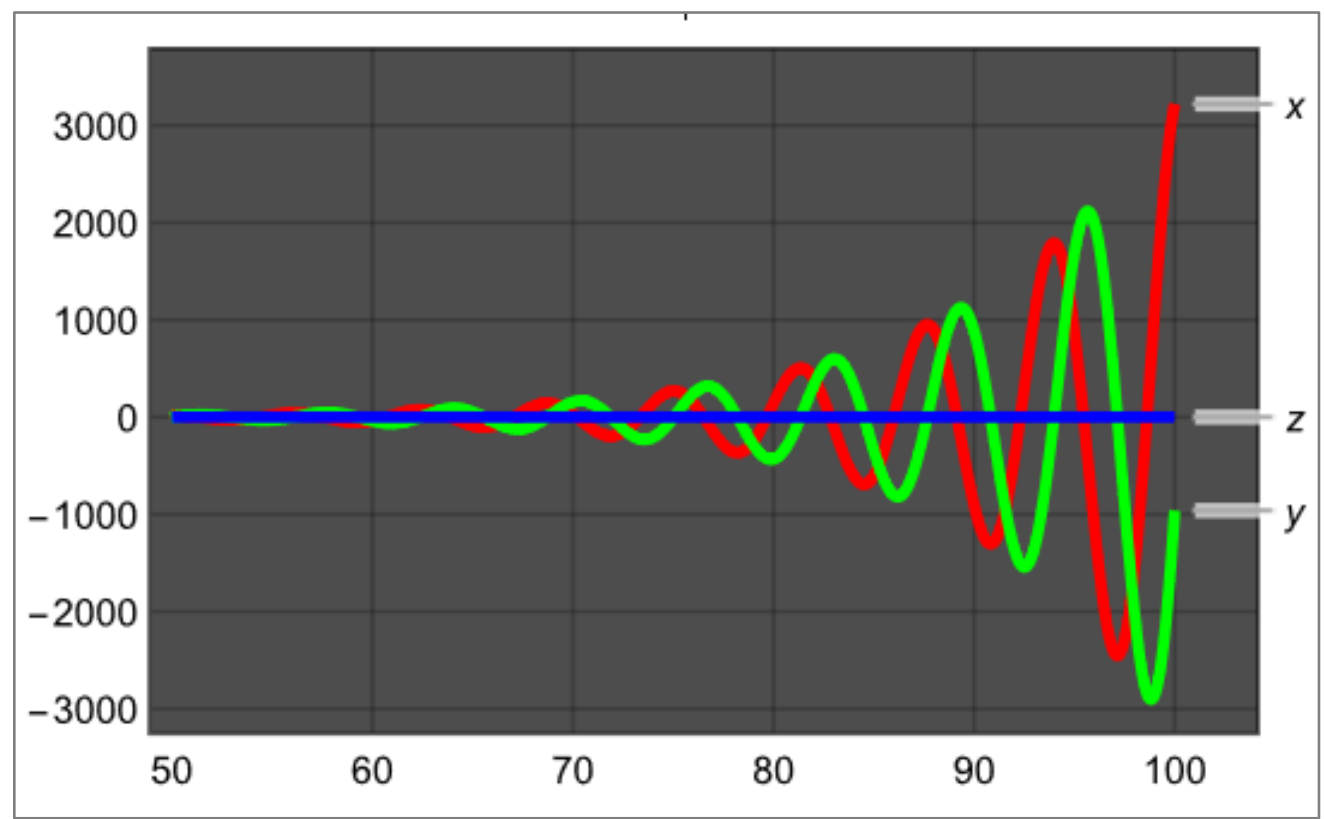

Рис. 2. Интегральные кривые 
2.1.3. Анализ и моделирование поведения системы в плоскости $x O y$

В этом случае система (2) упростится и пример вид

$\left\{\begin{array}{l}\frac{d x}{d t}=-y \\ \frac{d y}{d t}=x+a y\end{array} \quad a>0\right.$

Составим Якобиан для этой системы:

$J_{0}=\left(\begin{array}{cc}0 & -1 \\ 1 & a\end{array}\right)$.

Характеристическое уравнение $\left|\begin{array}{cc}-\lambda & -1 \\ 1 & a-\lambda\end{array}\right|=0$, значит имеется два собственных значения $\lambda_{1,2}=\frac{a \pm \sqrt{a^{2}-4}}{2}$.

В точке $O(0,0)$ при $a<2 \quad \lambda_{1,2}=\alpha \pm \beta i . \alpha>0$ и $\beta \neq 0$, значит в соответствии с классификацией особая точка будет являться неустойчивым фокусом.

В этом можно убедиться и другим методом (без нахождения собственных значений).

$\left|J_{0}\right|=1 ; \operatorname{tr}\left(J_{0}\right)=a>0$.

Так как $\left|J_{0}\right|>\left(\frac{\operatorname{tr}\left(J_{0}\right)}{2}\right)^{2}=\left(\frac{a}{2}\right)^{2} \quad$ то особая точка будет являться неустойчивым фокусом с траекториями в виде расходящихся логарифмических спиралей.

В нашем случае $\left.\frac{d y}{d t}\right|_{y=0}=x$, значит «раскручивание» траекторий происходит против часовой стрелки.

На рисунке (3) представлен фазовый портрет системы в плоскости $x O y$. 


\section{ИННОВАЦИОННЫЙ ДИСКУРС РАЗВИТИЯ СОВРЕМЕННОЙ НАУКИ И ТЕХНОЛОГИЙ}

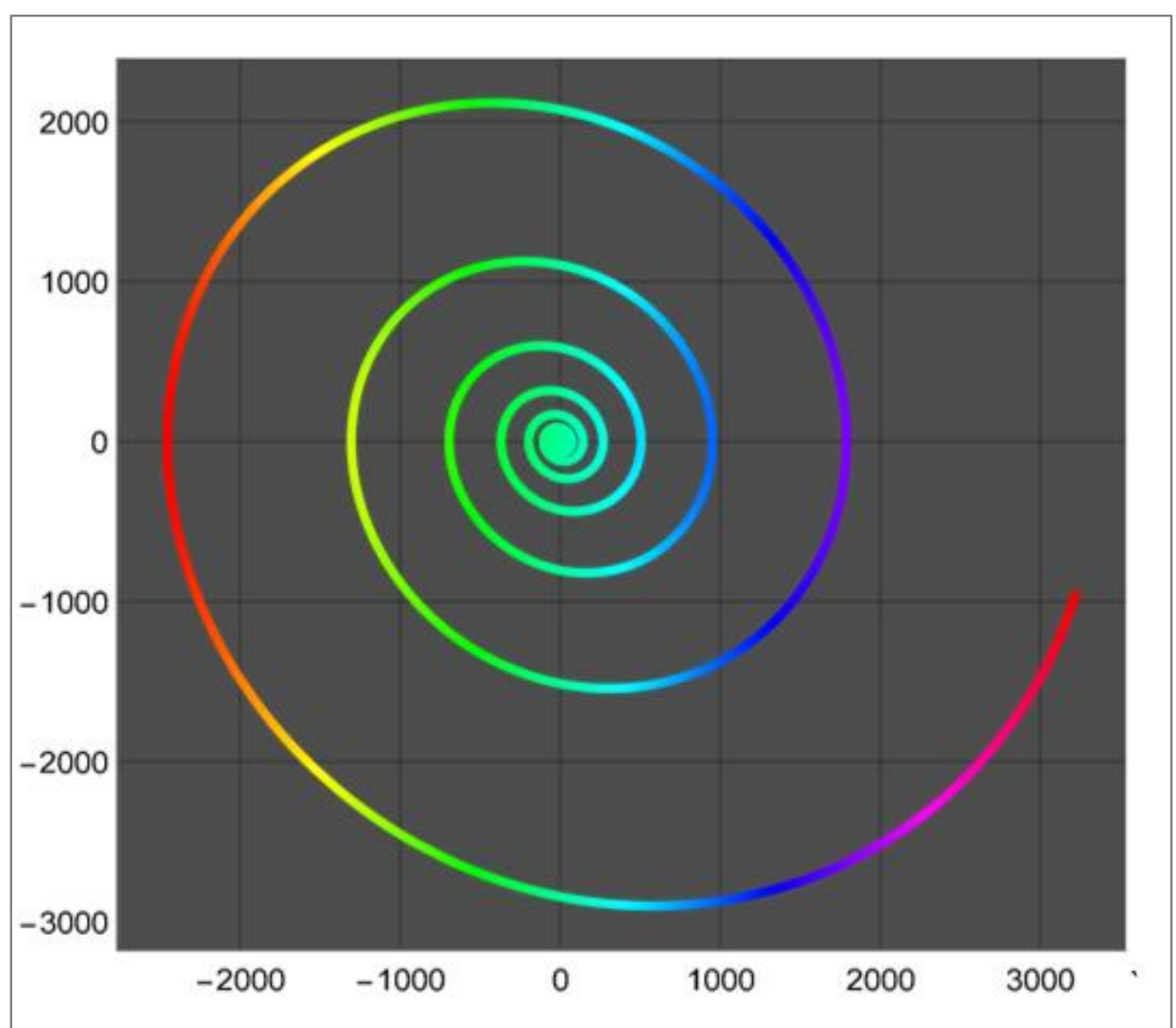

Рис. 3. Фазовая плоскость

2.2. Стационарная точка $E_{1}\left(c,-\frac{c}{a}, \frac{c}{a}\right)$

2.2.1. Основы качественного исследования

Аналог системы (4) для данной стационарной точки:

$$
\left\{\begin{array}{l}
\frac{d \tilde{x}}{d t}=-(\tilde{y}+\bar{z}) \\
\frac{d \tilde{y}}{d t}=\tilde{x}+a \tilde{y} \\
\frac{d \tilde{z}}{d t}=\frac{c}{a} \tilde{x}
\end{array}\right.
$$

Соответственно, определится якобиан, его определитель и след: 


$$
J_{1}=\left(\begin{array}{ccc}
0 & -1 & -1 \\
1 & a & 0 \\
\frac{c}{a} & 0 & 0
\end{array}\right),\left|J_{1}\right|=c ; \operatorname{tr}\left(J_{1}\right)=a
$$

Введя обозначения $p=-a, q=1+\frac{c}{a}, r=-c$ запишем характеристическое уравнение в виде:

$$
\lambda^{3}+p \lambda^{2}+q \lambda+r=0
$$

Корни этого уравнения определятся так:

$$
\begin{aligned}
& \lambda_{1}=-\frac{p}{3}-\frac{\sqrt[3]{2}\left(-p^{2}+3 q\right)}{3 \sqrt[3]{-2 p^{3}+9 p q-27 r+3 \sqrt{3} \sqrt{-p^{2} q^{2}+4 q^{3}+4 p^{3} r-18 p q r+27 r^{2}}}}+ \\
& +\frac{1}{3} \sqrt[3]{\frac{-2 p^{3}+9 p q-27 r+3 \sqrt{3} \sqrt{-p^{2} q^{2}+4 q^{3}+4 p^{3} r-18 p q r+27 r^{2}}}{2}} \\
& \lambda_{2,3}=-\frac{p}{3}+\frac{(1 \pm i \sqrt{3})\left(-p^{2}+3 q\right)}{32^{2 / 3} \sqrt[3]{-2 p^{3}+9 p q-27 r+3 \sqrt{3} \sqrt{-p^{2} q^{2}+4 q^{3}+4 p^{3} r-18 p q r+27 r^{2}}}} \\
& -\frac{(1 \mp i \sqrt{3})}{6} \sqrt[3]{\frac{-2 p^{3}+9 p q-27 r+3 \sqrt{3} \sqrt{-p^{2} q^{2}+4 q^{3}+4 p^{3} r-18 p q r+27 r^{2}}}{2}}
\end{aligned}
$$

2.2.2. Компьютерный эксперимент

В рассматриваемом случае система из окрестности стационарной точки $E_{1}\left(c,-\frac{c}{a}, \frac{c}{a}\right)$ достаточно быстро «скатывается» в зону притяжения нулевой стационарной точки $E_{0}(0,0,0)$ и далее ее поведение подчиняется закону, описанному ранее. На рисунках 4 и 5 представлено фазовое пространство и графики интегральных кривых для начальной эволюции системы. В дальнейшем при возрастании координаты $x$ происходит выход системы из плоскости $x O y$ (увеличение $z$ ). 


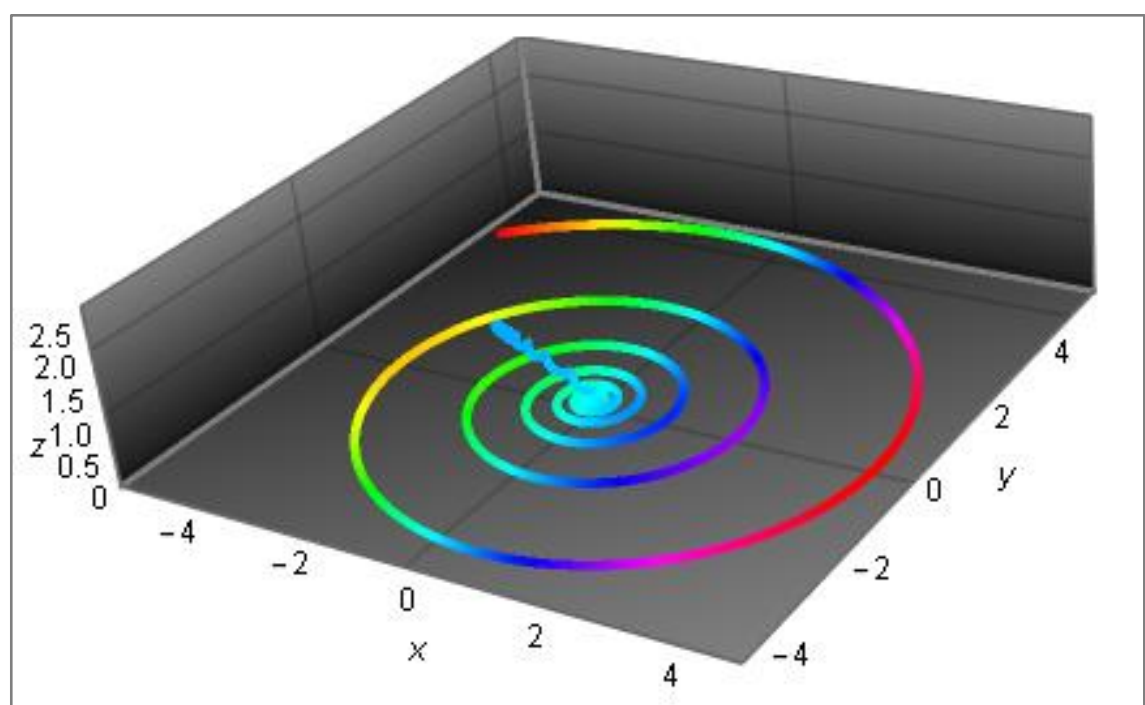

Рис. 4. Фазовое пространство

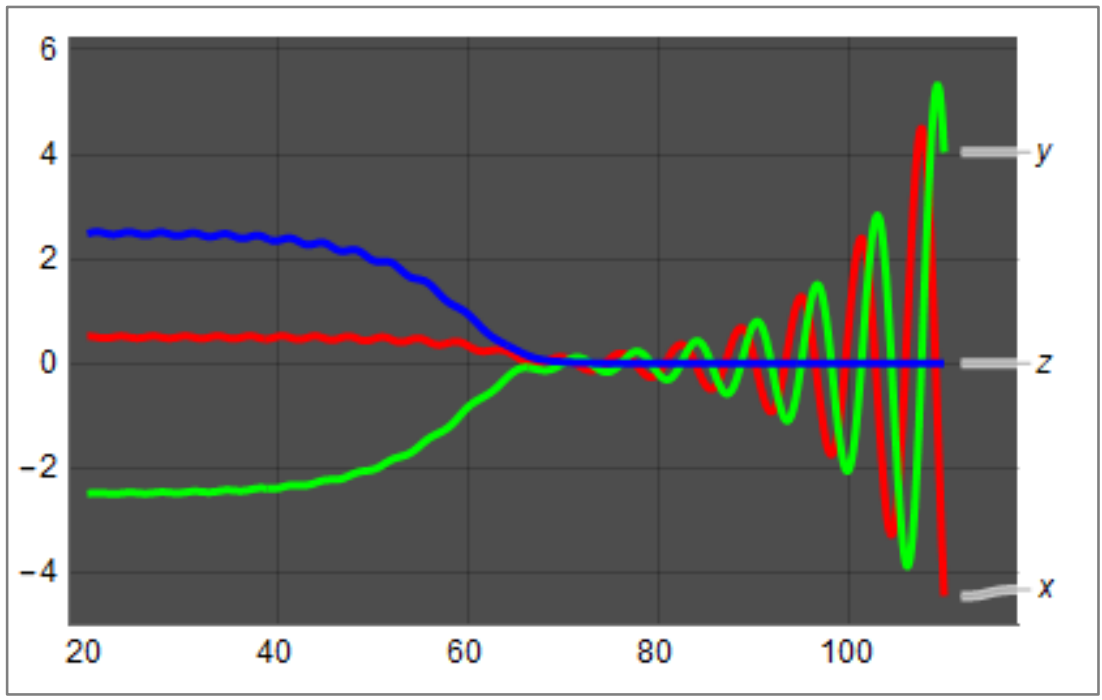

Рис. 5. Интегральные кривые

\section{3. Исследование классической модели.}

Классическая модель Рёсслера представлена системой уравнений (1).

$$
\left\{\begin{array}{l}
\frac{d x}{d t}=-(y+z) \\
\frac{d y}{d t}=x+a y \quad(a, b, c>0) \\
\frac{d z}{d t}=b+z(x-c)
\end{array}\right.
$$

Стационарные точки имеют координаты: 


$$
E_{1,2}\left(\frac{c \mp \sqrt{c^{2}-4 a b}}{2}, \frac{-c \pm \sqrt{c^{2}-4 a b}}{2 a}, \frac{c \mp \sqrt{c^{2}-4 a b}}{2 a}\right)
$$

На рисунке 6 представлена траектория системы в фазовом пространстве при $a=0.2 . b=0.2, c=5$.

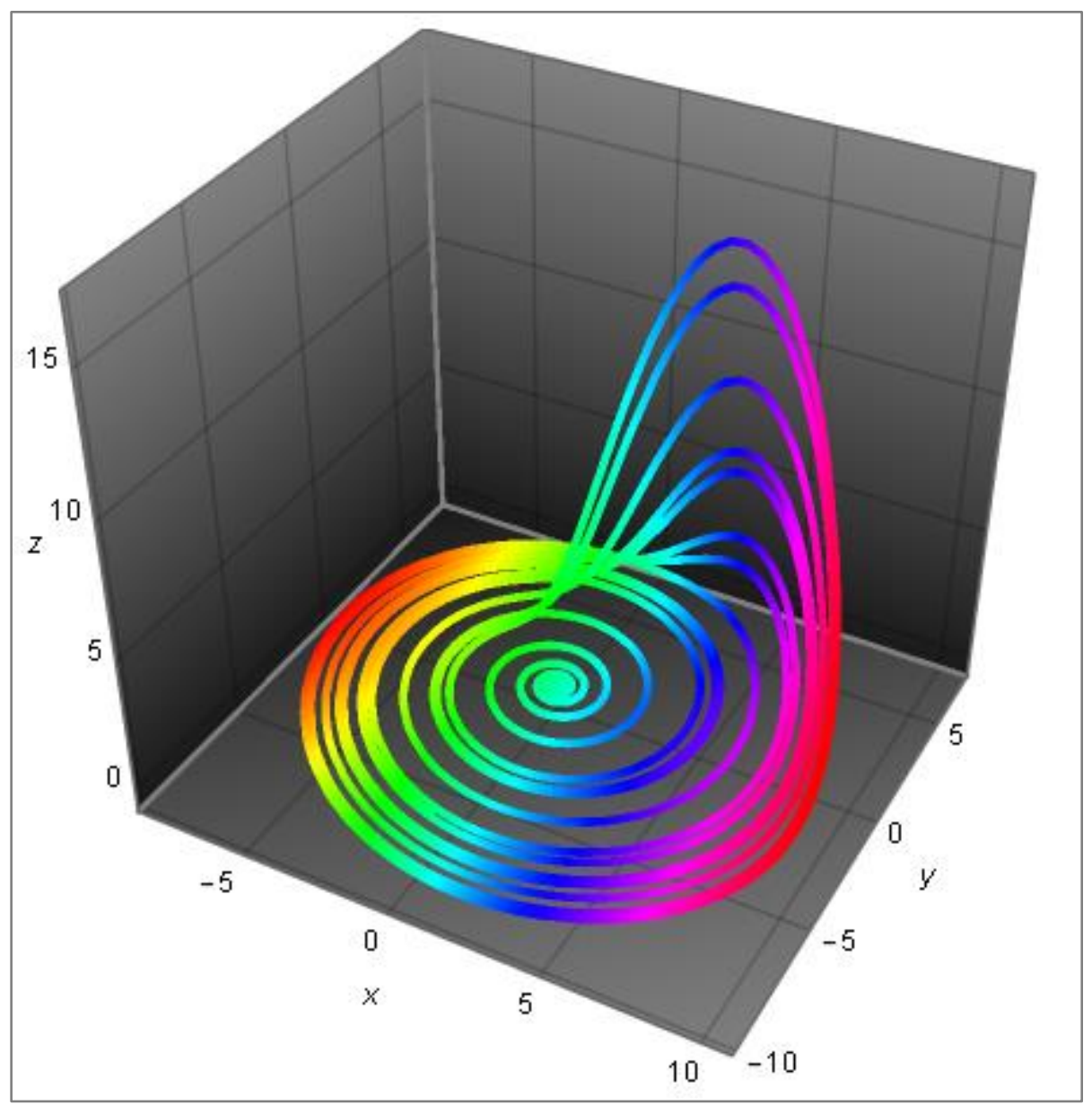

Рис. 6. Фазовое пространство системы при с=5

При данных значениях параметров система классифицируется как хаотический аттрактор.

Решения системы дифференциальных уравнений $x=x(t), y=y(t), z=z(t)$ представлены на рисунке 7 . 


\section{ИННОВАЦИОННЫЙ ДИСКУРС РАЗВИТИЯ

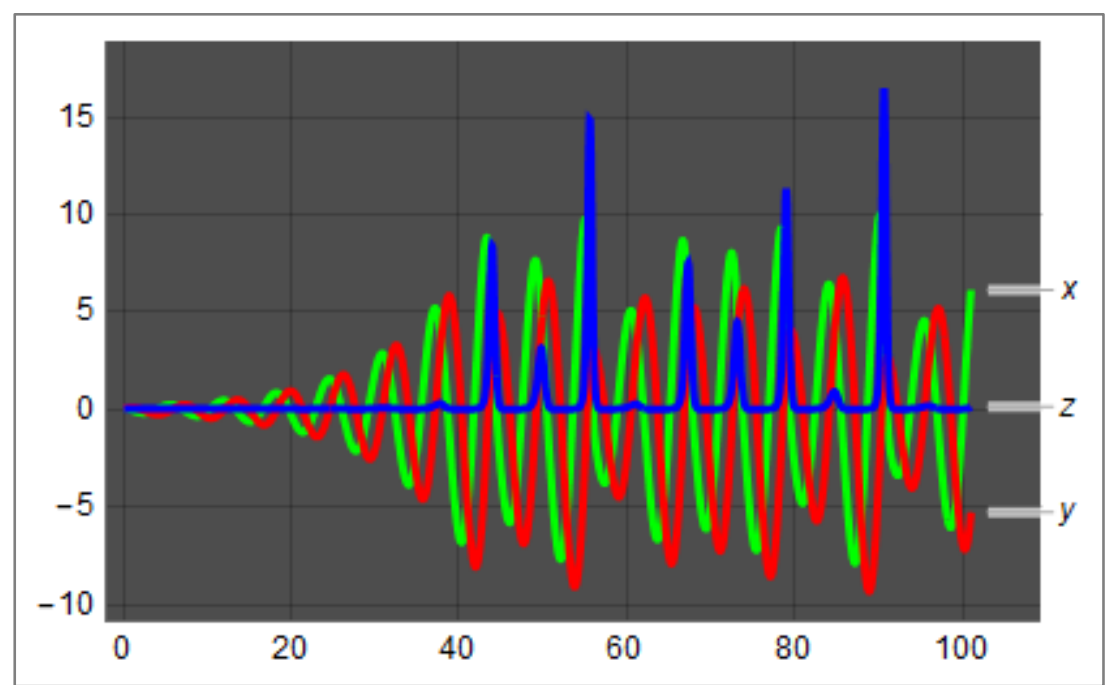

Рис. 7. Интегральные кривые

Рисунок 8 демонстрирует поведение системы в плоскости хОу.

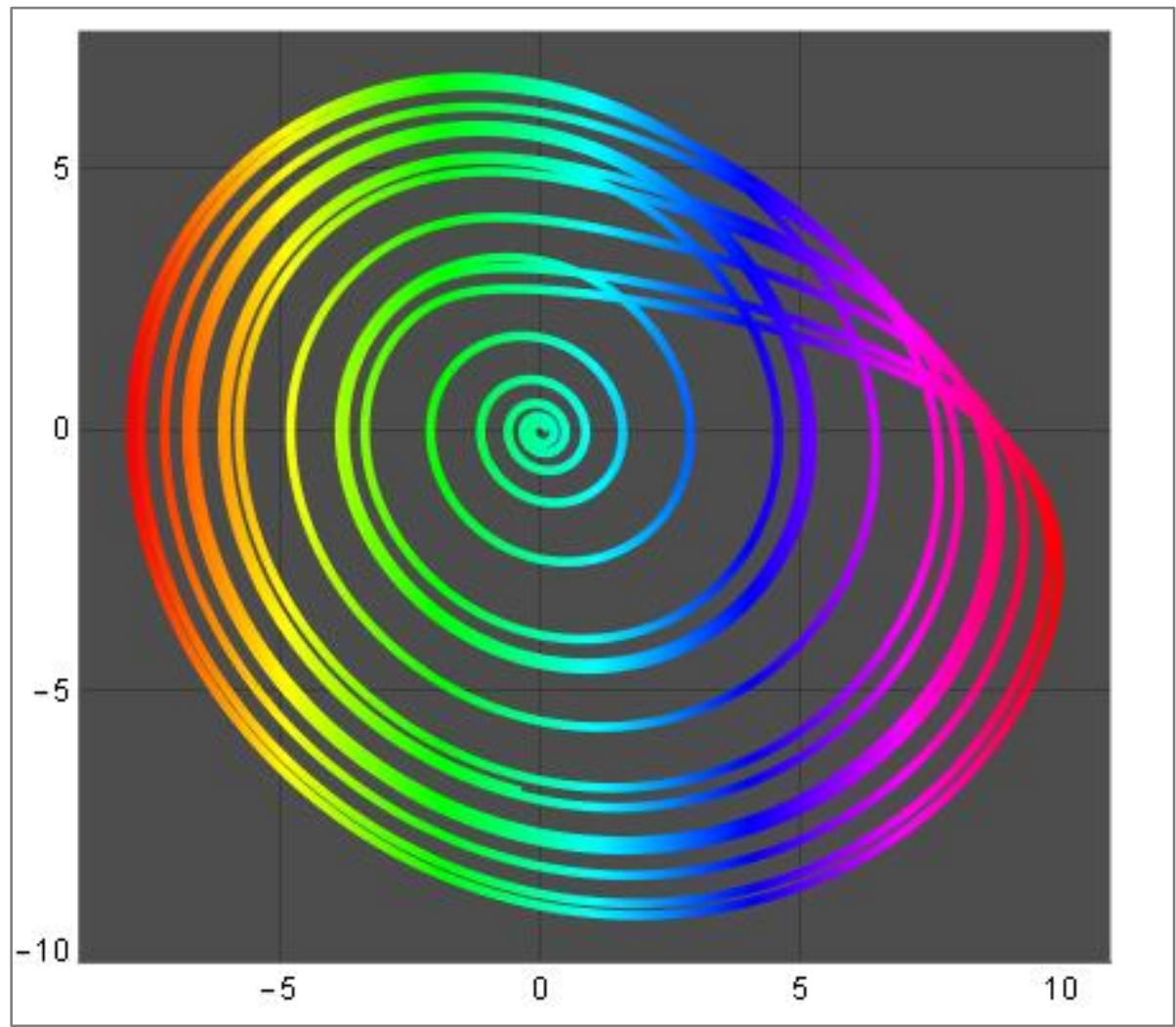

Рис. 8. Траектории в фазовой плоскости

Параметр $c$ является ключевым, например при $c=3$ (при неизменном значении величин остальных параметров) поведение системы определяется устойчивыми предельными циклами (см. рисунок 9) 


\section{ИННОВАЦИОННЫЙ ДИСКУРС РАЗВИТИЯ \\ СОВРЕМЕННОЙ НАУКИ И ТЕХНОЛОГИЙ}

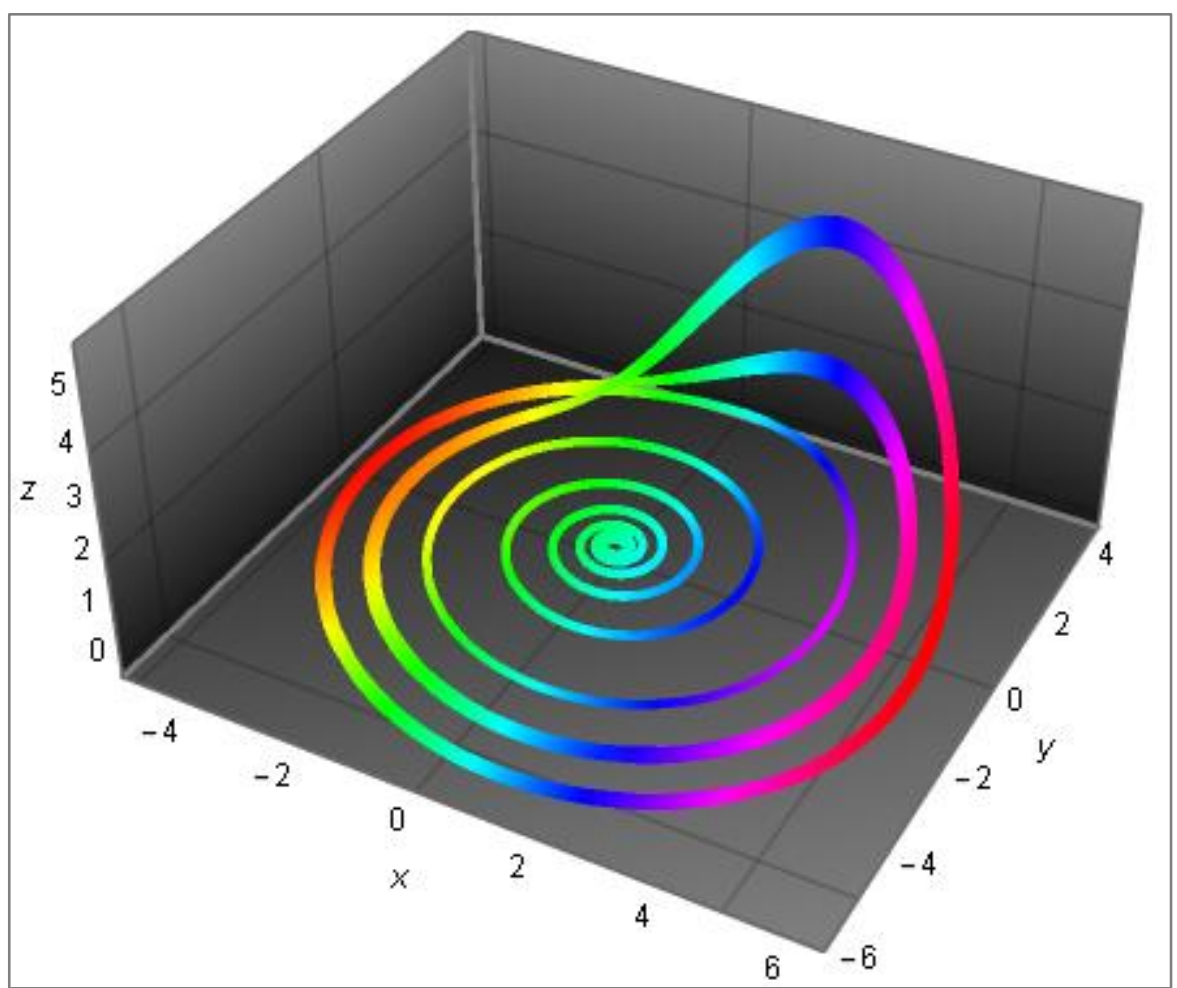

Рис. 9. Траектории в виде устойчивых предельных циклов

\section{4. Исследование обобщенной модели Рёсслера.}

В системе (3) имеются две стационарные точки:

$E_{0}(0,0,0), E_{1}\left(c-a b, \frac{a b-c}{a}, \frac{c-a b}{a}\right)$.

Исследуем поведение системы в окрестности точки $E_{0}(0,0,0)$.

Якобиан и его характеристики:

$J_{0}=\left(\begin{array}{ccc}0 & -1 & -1 \\ 1 & a & 0 \\ b & 0 & -c\end{array}\right),\left|J_{0}\right|=2 a-c, \operatorname{tr}\left(J_{0}\right)=a-c$.

Корни соответствующего характеристического уравнения находятся по формулам (4) при $p=c-a, q=b+1-a c, r=c-a b$

На рисунке 10 представлены результаты моделирования при $a=0.2, b=2.0, c=1.0$. Траектория из точки $\left(x_{0}, y_{0}, z_{0}\right)$ притягивается к стационарной точке $E_{0}(0,0,0)$ и в ней остается. 


\section{ИННОВАЦИОННЫЙ ДИСКУРС РАЗВИТИЯ \\ СОВРЕМЕННОЙ НАУКИ И ТЕХНОЛОГИЙ}

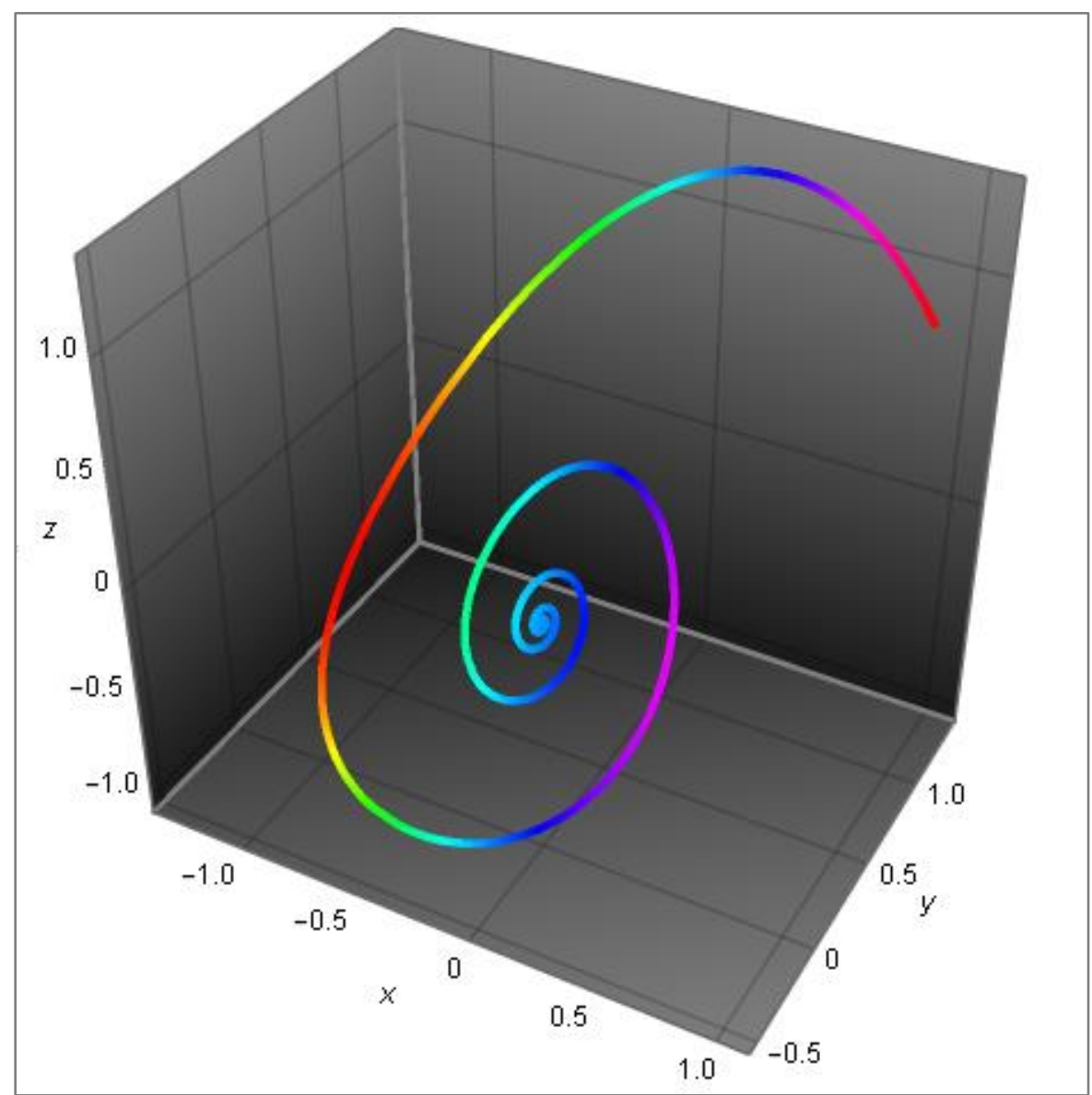

Рис. 10. Фазовое пространство

Соответствующие интегральные кривые при заданных параметрах моделирования представлены на рисунке 11.

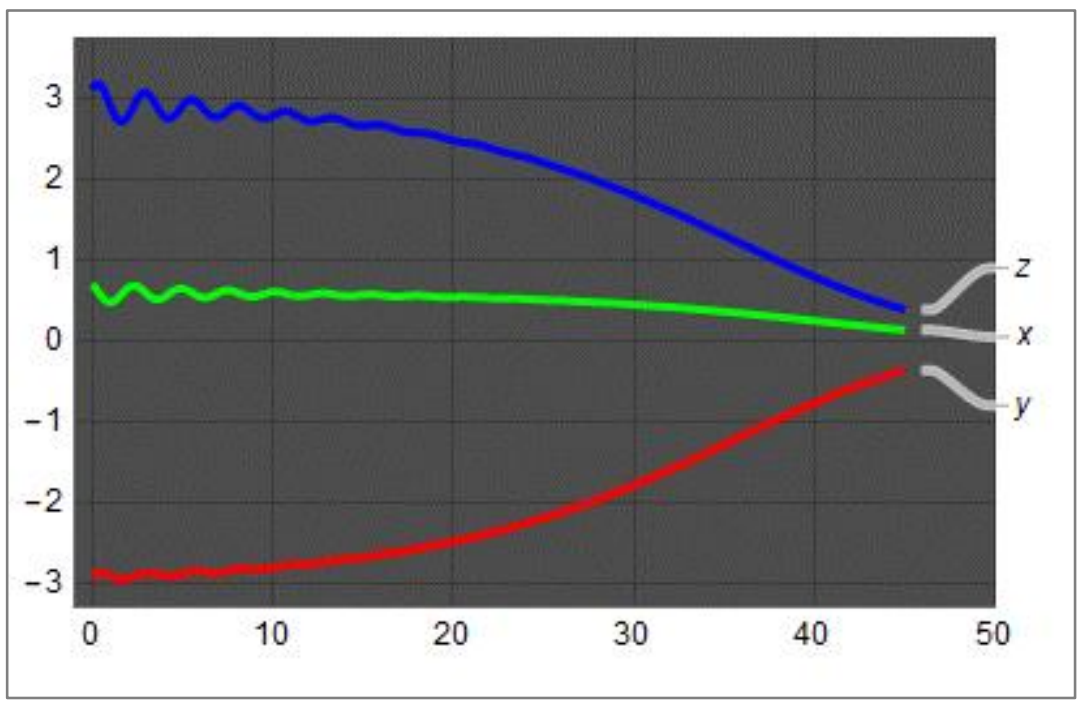

Рис. 11. Интегральные кривые 
Анализ поведения системы в плоскости $x O y$ свидетельствует о том, что $E_{0}(0,0,0)$ является стационарной точкой типа фокус. Фазовая траектория в виде спирали закручивается против часовой стрелки.

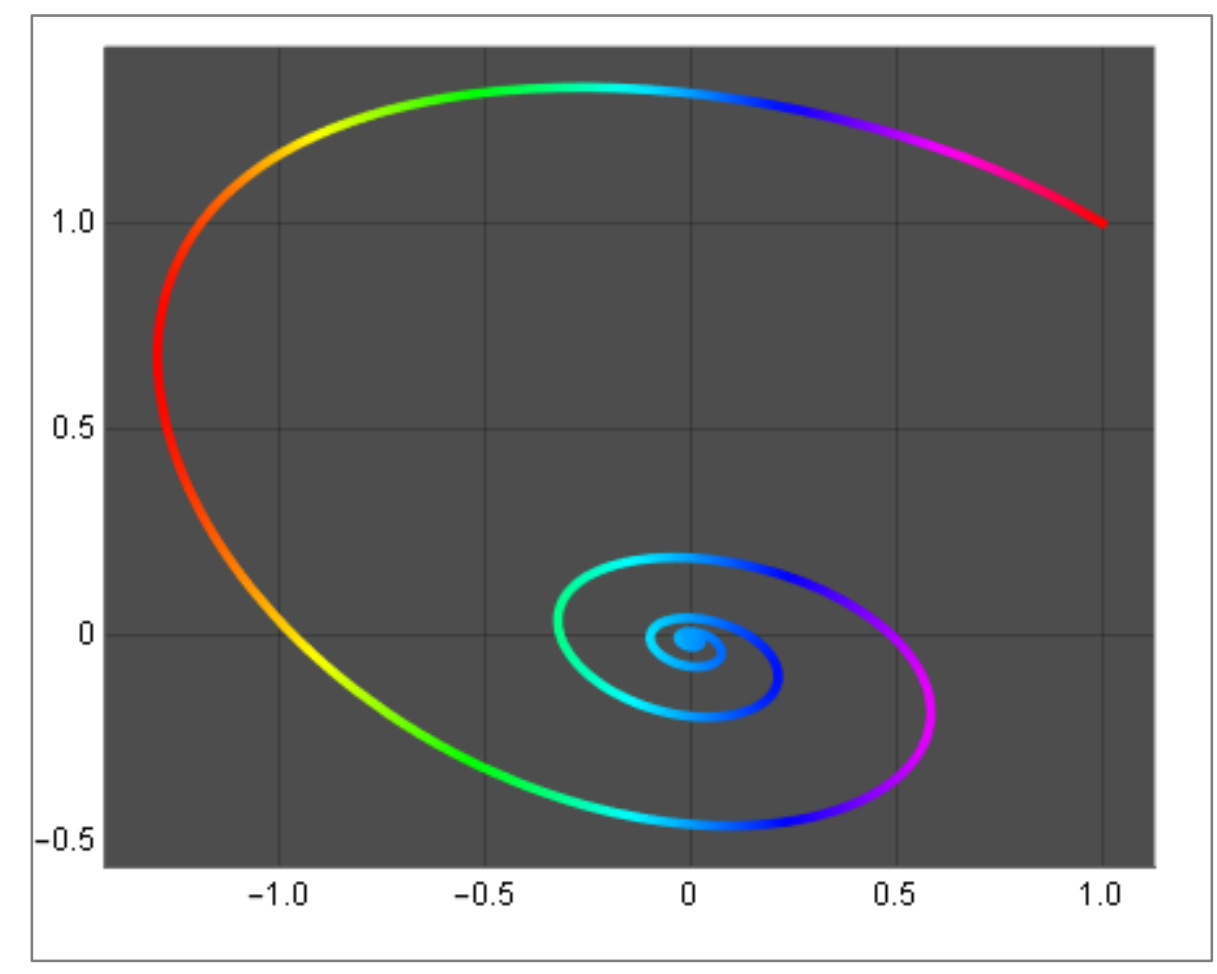

Рис. 12. Фазовая плоскость $x O y$

По той же схеме исследуется поведение обобщенной системы Рёсслера в окрестности второй особой точки $E_{1}\left(c-a b, \frac{a b-c}{a}, \frac{c-a b}{a}\right)$

\section{Заключение.}

Проверочные вычислительные эксперименты и практическая апробация выполнены на базе аналитической платформы компьютерной алгебры Wolfram Mathematica, являющейся наиболее современным программным комплексом символьной и вычислительной математики, поддерживающим все актуальные парадигмы компьютерного моделирования, машинного обучения и разработки программного обеспечения систем искусственного интеллекта [2].

Настоящее исследование выполнено в продолжение работ по анализу сложных диссипативных структур со странными аттракторами [3]. 


\section{Список литературы}

1. Игорь Н. фон Бекман Нелинейная динамика сложных систем: теория и практика. URL: http://profbeckman.narod.ru/NelDin/NelDinText2.pdf (дата обращения 08.05.2021).

2. Stephen Wolfram. An Elementary Introduction to the Wolfram Language. URL: https://www.wolfram.com/language/elementary-introduction/2nd-ed/ (Дата обращения 08.05.2021).

3. Осипов Г.С. Исследование динамической системы Лоренца в среде имитационного моделирования AnyLogic // Инновационное развитие науки: фундаментальные и прикладные проблемы: Монография - 2020. - С. 248-259.

Г.С. Осипов, Н.С. Вашакидзе, Г.В. Филиппова, Н.Л. Рауш, 2021 